\title{
A ELABORAÇÃO DE CENÁRIOS ESTRATÉGICOS COMO VANTAGEM COMPETITIVA
}

\section{THE ELABORATION OF STRATEGIC SCENARIOS AS COMPETITIVE ADVANTAGE}

\section{DOl: http://dx.doi.org/10.21714/raunp.v10i1.1762}

Wanderson F. M. de Oliveira

Mestre em Administração. Professor da Universidade Potiguar. E-mail: wandersonf81@gmail.com

\section{Walid Abbas El-Aouar}

Doutor em Administração. Professor da Universidade Potiguar. E-mail: walidbranco@gmail.com

\section{Kléber Cavalcanti Nóbrega}

Doutor em Engenharia de Produção. Professor da Universidade Potiguar. E-mail: klebercnobrega@gmail.com

Envio em: Novembro de 2017

Aceite em: Dezembro de 2017

\section{RESUMEN}

Este estudio ha tenido, como objetivo, proponer una reflexión acerca de como los temas "elaboración de escenarios" y "ventaja competitiva" se plantean en la literatura, buscando demostrar evidencias de como están relacionados y dar proposición para investigaciones que averigüen a fondo esa relación. En cuanto al método, la investigación presenta el enfoque cualitativo, siendo la recolección de los datos de fuente secundaria, por medio de artículos obtenidos de las bases de investigación Ebsco Host, Emerald, ProQuest, Science Direct e Scopus, objetivando la realización de estudio bibliométrico y bibliográfico. Los resultados sugieren que, en un mercado de competencia globalizada, en un ambiente de constantes cambios y de incertidumbres en lo que concierne al presente y al futuro, las organizaciones necesitan desarrollar su competitividad y que la elaboración de escenarios estratégicos concede a la empresa ventaja competitiva, una vez que favorece la elaboración de la Estrategia Robusta. El resultado nos ha conducido a la conclusión de que la construcción de escenarios es un instrumento que posibilita a la empresa identificar oportunidades y amenazas en el ambiente externo, para planificar un mejor aprovechamiento de las oportunidades y minimizar las amenazas, siendo, por lo tanto, un instrumento potenciador de las estrategias para la sobrevivencia y desarrollo de la organización.

Palabras clave: Escenarios Estratégicos. Ventaja Competitiva. Planificación Estratégico. Competitividad. Estrategia Competitiva

\section{ABSTRACT}

This study had as objective to propose a reflection on the themes "elaboration of scenarios" and "competitive advantage" have been worked out in the literature, seeking to demonstrate evidence of how are related and provide research proposition to investigate thoroughly. As for the method, the search features of qualitative approach, being the secondary source data collection, through articles extracted from Ebsco Host research databases, Emerald, ProQuest, Science Direct and Scopus, aiming at the realization of bibliographic and bibliometric study. The results suggest that, in a globalized, competitive market in an environment of constant change and uncertainty about the present and the future, organizations need to develop their competitiveness and the development of strategic scenarios gives the company a competitive advantage, since it favours the elaboration of Robust Strategy. It was concluded that the construction of scenarios is an instrument that enables the company to identify opportunities and threats in the external environment, to plan better use of opportunities and minimize threats, being therefore a catalyzing instrument of strategies for survival and development of the organization.

Keywords: Strategic Scenarios. Competitive Advantage. Strategic Planning. Competitiveness. Competitive Strategy 


\section{INTRODUÇÃO}

Com a aceleração das mudanças em um mundo globalizado, torna-se cada vez mais perceptível para as organizações a necessidade de se adequarem a essas mudanças, por questão de manutenção e crescimento, considerando-se que as certezas acerca das tendências conjunturais do presente são ilusórias e os administradores necessitam buscar uma compreensão mais apurada da realidade e dos riscos (SCHWARTZ, 2004; WRIGHT; SPERS, 2006). Nessa perspectiva, o pensamento e a administração estratégica convertem-se importantes para a sobrevivência das empresas, conforme ocorre a evolução dos processos de descontinuidade do ambiente e da dificuldade de previsibilidade (FALLER; DE ALMEIDA, 2014; MÜLLER, 2003; PIZZINATTO et al., 2010; VICENTINE, 2009).

As empresas são sistemas abertos que recebem influências externas do ambiente, que podem afetar seu funcionamento e seu desempenho (SERRA; TORRES; TORRES, 2004). Assim, a análise ambiental ajuda a identificar tendências e serve como base para diagnóstico de cenários futuros. Previsões bem elaboradas auxiliam no planejamento e na tomada de decisões e favorecem os executivos a descobrir e a avaliar oportunidades e ameaças. Alinhado a essa perspectiva, Schwartz (2004) afirma que os cenários auxiliam na organização das percepções dos gestores, e, identificar um cenário não tem como objetivo escolher um futuro preferido e aguardar que ele se realize, nem tão pouco é encontrar o futuro mais provável e se adaptar a ele. Em vez disso, a elaboração de cenários objetiva tomar decisões estratégicas que sejam plausíveis para todos os futuros possíveis. Conforme sublinha Fotr et al. (2014), a gestão estratégica nas empresas modernas tem de lidar com ambientes de negócios de forma dinâmica em mudança. Enquanto as empresas desejarem gerenciar com sucesso seu futuro, reforçar a sua competitividade e gerar valor para os acionistas, eles devem ser capazes de prever o aparecimento de fatores de risco que podem afetar o desempenho da empresa, o qual resulta de um plano estratégico.

Segundo Magalhães e Barp (2014), para desempenharem suas atribuições os governos, as empresas e até mesmo os indivíduos, necessitam utilizar instrumentos de diversas naturezas de forma eficaz, objetivando maximizar os retornos sociais e econômicos que visam tanto o bem-estar da sociedade como de si próprios. Entretanto, para que isso ocorra, afirmam os autores, o processo de tomada de decisões deve ser coordenado por meio de técnicas que permitam eleger e relacionar as melhores alternativas de ação tendo como referência a melhor relação entre custo e benefício. Entre essas técnicas, encontra-se a elaboração de cenários estratégicos (MAGALHÃES; BARP, 2014).

Desse modo, este ensaio objetiva propor uma reflexão acerca de como os temas "elaboração de cenários" e "vantagem competitiva" vêm sendo sistematizados na literatura, buscando demonstrar evidências de como estão relacionados e fornecer proposição para pesquisas que investiguem a fundo essa relação.

A relevância deste trabalho pode ser considerada em função de que, por causa da concorrência globalizada e diante das constantes mudanças da conjuntura presente e dos riscos e incertezas do futuro para que as organizações sobrevivam e cresçam, o desenvolvimento da competência na elaboração de cenários estratégicos torna-se vantagem competitiva.

\section{PROCEDIMENTOS METODOLÓGICOS}

Os procedimentos metodológicos adotados seguem os padrões da pesquisa teórica, a qual requer que seja construída de forma sistemática e rigorosa, condição indispensável para credibilidade dos resultados assinalados no trabalho, bem como para a formação de conhecimento em uma base sólida a partir do mesmo (ABREU; ALCÂNTARA, 2014; TRANFIELD; DENYER; SMART, 2003; WEBSTER; WATSON, 2002).

Assim, optou-se por uma pesquisa qualitativa, a qual auxilia nas definições do problema da pesquisa, bem como justifica a necessidade da realização do estudo (SAMPIERI; COLLADO, LÚCIO, 2013; SNYDER, 2012) e demonstra a verdade não por meio de dados quantitativos ou numéricos (como ocorre na pesquisa quantitativa), mas na forma de argumentação estruturada, lógica e bem fundamentada das ideias (MICHEL, 2015).

A coleta dos dados foi secundária, uma vez que os artigos foram extraídos das bases de pesquisa reconhecidas como de referência no mundo acadêmico (HARZING; ALAKANGAS, 2015), apresentadas no Quadro 2, visando à realização de estudo bibliométrico e bibliográfico, pois permitem examinar uma variedade de assuntos para ajudar a entender, organizar e explorar o que já foi feito ou pesquisado a partir das fontes bibliográficas (ALVES; OLIVEIRA, 2014; FERREIRA, 2011). A pesquisa nas fontes de dados foi realizada com os parâmetros listado no Quadro 1, os quais foram utilizados para ambos os estudos. 
QUADRO 1: PARÂMETROS DE PESQUISA.

\begin{tabular}{|c|c|c|c|}
\hline $\begin{array}{l}\text { BASE DE } \\
\text { PESQUISA }\end{array}$ & $\begin{array}{l}\text { GRANDE } \\
\text { AREA }\end{array}$ & $\begin{array}{l}\text { LINHA DE } \\
\text { PESQUISA }\end{array}$ & $\begin{array}{l}\text { MATERIAL } \\
\text { PESQUISADO }\end{array}$ \\
\hline Ebsco Host & $\begin{array}{l}\text { Bussiness; Economics } \\
\text { Social Sciences \& } \\
\text { Humanities }\end{array}$ & $\begin{array}{l}\text { Accounting \& Finance; Economics; HR \& } \\
\text { Organizational Behaviour; } \\
\text { Information \& Knowledge; Management; } \\
\text { Management Science \& Operations; Marketing; } \\
\text { Property Management \& Built Environment; Public } \\
\text { Policy \& Environmental Management; Strategy }\end{array}$ & $\begin{array}{l}\text { Journals, books, } \\
\text { review, conference } \\
\text { paper and article }\end{array}$ \\
\hline Emerald & $\begin{array}{l}\text { Bussiness; Economics } \\
\text { Social Sciences \& } \\
\text { Humanities }\end{array}$ & $\begin{array}{l}\text { Accounting \& Finance; Economics; Information \& } \\
\text { Knowledge; Management; Management Science } \\
\text { \& Operations; Marketing; Management \& Built } \\
\text { Environment; Strategy }\end{array}$ & $\begin{array}{l}\text { Journals, books, } \\
\text { review and article }\end{array}$ \\
\hline ProQuest & Social Sciences & Applied Social Sciences Index \& Abstracts (ASSIA) & $\begin{array}{l}\text { Journals, books, } \\
\text { review and article }\end{array}$ \\
\hline Science Direct & $\begin{array}{l}\text { Bussiness; Economics } \\
\text { Social Sciences \& } \\
\text { Humanities }\end{array}$ & $\begin{array}{l}\text { Business, Management and Account; Social } \\
\text { Sciences; Economics, Econometrics and Finance; } \\
\text { Decision Sciences; Environmental Science }\end{array}$ & $\begin{array}{l}\text { Journals, books, } \\
\text { review and article }\end{array}$ \\
\hline Scopus & $\begin{array}{l}\text { Social Sciences \& } \\
\text { Humanities }\end{array}$ & $\begin{array}{l}\text { Business, Management and Account; Social } \\
\text { Sciences; Economics, Econometrics and Finance; } \\
\text { Decision Sciences; Environmental Science }\end{array}$ & $\begin{array}{l}\text { Journals, books, } \\
\text { review and article }\end{array}$ \\
\hline
\end{tabular}

Fonte: Elaborado pelos autores.

Primeiramente foram pesquisados artigos publicados no período de 2000 a 2016, utilizando-se, para tanto, das palavras-chave relacionadas ao tema deste trabalho, sendo elas: strategic scenarios, competitive advantage, strategic planning, competitiveness $e$ competitive strategy. Para efetivar a triagem dos artigos, filtrou-se nas fontes de pesquisa que as publicações fossem organizadas por ordem de relevância ou de citação.

Posteriormente, foi realizada a triagem dos artigos para a pesquisa bibliográfica, os quais foram selecionados pelos critérios de: (1) maior convergência de conteúdo para com este estudo; (2) artigos terem sido bastante citados; e, (3) serem de relevância para a temática. Para a etapa bibliométrica, a contabilização dos trabalhos foi feita no período de 5 a 8 de junho de 2016 e, para a bibliográfica, ela ocorreu no período de junho a setembro de 2016. Os resultados dos dados coletados nas bases de pesquisa serão discutidos na Seção4.

\section{CENÁRIOS: CONCEITOS E IMPORTÂNCIA}

O termo "cenário" é rico de significados, variando de sentido em função do campo de especialização, as- sumindo conotações desde montagens de ambientes para filmagens, até combinações estatísticas sobre as tendências macroeconômicas (COSTA et al., 2007). $\mathrm{Na}$ literatura podem ser encontrados diversos conceitos para cenários. Um exemplo é o de Schenatto et al. (2011), os quais afirmam que em termos conceituais, cenários são utilizados como o estudo do futuro. Carlos et al. (2013) entendem que a visão do futuro avançou de uma abordagem profética e de ensaios de futurologia e assimilou a ciência, adotando técnicas numéricas de previsão.

Segundo Gugan (2008), cenários oferecem visões alternativas do futuro. Eles identificam algumas diferenças básicas e os principais intervenientes, bem como a sua motivação e oferta diferentes perspectivas sobre o desenvolvimento mundial futuro. A elaboração e a aplicação de cenários podem ajudar a encontrar a maneira como enfrentar as incertezas futuras. Para Oliveira (2014, p. 330), "Cenários representam situações, critérios e medidas para a preparação do futuro da empresa". Já Porter (2004) afirma que cenário é uma visão internamente consistente do que o futuro pode ser, e tem como principais funções a avaliação do que foi planejado, o apoio à elaboração de objetivos e de estratégias, a análise de alternativas, o estímulo à criatividade e a preparação para enfrentar 
descontinuidades.

Porter (2004), Prahalad e Hamel (2005) e Ribeiro (2012), observam que, quando se utiliza o planejamento por cenários, é obtida uma vantagem em relação às tradicionais formas de planejamento, considerando-se que esse tipo de planejamento é integrado às incertezas na construção do futuro, bem como à variedade de possibilidades, organizando-as em narrativas de simples entendimento. Ademais, Ribeiro (2012) faz alusão à utilização do planejamento por cenários para: (1) identificar possíveis oportunidades de atuação; (2) testar a estratégia em múltiplos cenários; (3) monitorar a execução da estratégia; (4) pesquisar mudanças no ambiente para determinar as estratégias que deverão ser adaptadas ou alteradas para a sobrevivência da organização; e (5) reduzir as incertezas com relação à capacidade da liderança em promover ajustes.

Os cenários são elaborados para auxiliar na formulação de uma estratégia e trazer qualidade de previsão para os principais indicadores de desempenho, relacionando-os com as percepções de causa e efeito (BUYTENDIJK; HATCH; MICHELI, 2010; POSTMA; LIEBL, 2005). Onkal, Sayim e Gönül (2013) apresentam como os cenários podem ser utilizados para auxiliar a avaliação quantitativa na previsão. Eles apontam os efeitos da elaboração de cenários como sendo importantes no planejamento e sugerem que os cenários alternativos podem embasar os decisores na consideração de resultados sobre os futuros alternativos. Ademais, como ressaltado a Central Intelligence Agency (2006), os cenários proporcionam uma visão mais dinâmica acerca das possibilidades futuras e chamam a atenção sobre as interações que podem ser significativas no estabelecimento das estratégias.

Assim, entende-se por cenário a elaboração das possibilidades do futuro, o qual auxilia o planejamento da estratégia para melhor enfrentá-lo, conferindo a empresa vantagem competitiva diante da concorrência. Para tanto, na próxima Seção, serão expostas formas e os cuidados que se deve ter para a elaboração de cenários.

\section{MONITORAMENTO AMBIENTAL E ELABORAÇÃO DE CENÁRIOS}

Blois e Sousa (2008) e Wright e Spers (2006) pontuam que a elaboração de cenários não é para "acertar" previsões do futuro, mas sim para melho- rar a base de informações e a compreensão acerca das decisões que necessitam ser tomadas no presente para assegurar objetivos futuros. Por sua vez, James (2002) advoga que, ao se analisar o passado, constata-se a ocorrência de mudanças, rupturas e descontinuidades nas estruturas sociais e econômicas e isso serve de alerta para que seja evitada a prática de projetar o futuro simplesmente como uma extrapolação de um período de tempo do presente.

Para que os cenários sejam elaborados, é necessário que seja considerado o conjunto de forças que atuam sobre o sistema em estudo (PALEO, 2006; DA SILVA; SPERS; WRIGHT, 2012; WRIGHT; KROLL; PARNELL, 2009). Já para Schoemaker (1995), ao contemplar o futuro, é útil considerar três classes de conhecimento: 1) Coisas que sabemos que sabemos; 2) Coisas que sabemos que não sabemos; e 3) Coisas que não sabemos que não sabemos.

Segundo Friedman (2009), geopolítica é uma forma de se pensar sobre o mundo de hoje e de prevê os cenários que ocorrerão mais adiante e o termo aplica o conceito de mão invisível (de Adam Smith) ao comportamento das nações e de outros atores internacionais. O referido autor ressalta, ainda, que tanto a geopolítica como a economia partem do pressuposto que os jogadores (nações e atores internacionais) são racionais e que buscam o melhor para si, explicando, assim, pelo menos em grande parte, as mudanças observadas na sociedade e as incertezas do futuro.

Por outro lado, Godet (2000) e Vianello e Teixeira (2012) sugerem que não há um único método para a elaboração de cenários, mas uma variedade de métodos para a construção, sendo alguns mais simples, outros mais sofisticados. Complementando essa perspectiva, Coates (2000) sustenta que os cenários devem ser desenvolvidos a partir da identificação e definição do universo alvo, da definição das variáveis que serão importantes para projetar o futuro, da identificação dos temas para os cenários e da criação dos cenários.

Para Brandalise et al. (2012) e Ribas (2008), a simulação de cenários é uma ferramenta que auxilia na ordenação da percepção em relação aos contextos futuros, nos quais as decisões tomadas no presente terão consequências adiante. Assim, os cenários poderão servir na arquitetura dos pilares estratégicos que facilitarão o alcance dos objetivos organizacionais. Em linha semelhante, Van der Heijden (2009) complementa, ao afirmar que a visualização de cenários múltiplos serviria como base de testes de políticas e de planos das empresas e o objetivo é desenvolver 
projetos com probabilidades de retornos positivos sob qualquer um dos cenários.

Bontempo (2000) fez a comparação de diversas técnicas de elaboração de cenários e utilizou um quadro para comparar as etapas em cada uma das técnicas. As etapas descritas nos quadros são: 1) identificar as decisões principais; 2) relacionar variáveis de impacto, 3) analisar as variáveis; 4) extrapolar tendências; 5) analisar impacto cruzado; 6) preparar cenários iniciais; 7) realizar a análise de sensibilidade; 8) construir cenários detalhados; 9) analisar as implicações dos cenários; e 10) monitorar o ambiente.

De acordo com Rojo (2010), a simulação de cenários se constitui em uma etapa importante que antecede ao planejamento estratégico. Leão et al. (2010) e Oliveira (2012) sugerem que na elaboração de cenários seja construída uma visão panorâmica do futuro, por meio da apresentação de sequências de eventos ou de cenas, desdobradas a partir do presente, até um horizonte temporário definido, constando de interações entre variáveis interdependentes e dimensões estratégicas, conforme apresentadas no Quadro 4.

QUADRO 4: VARIÁVEIS INTERDEPENDENTES

\begin{tabular}{|c|c|c|c|c|}
\hline ECONÔMICA & TECNOLÓGICA & POLÍTICO & SOCIOCULTURAL & DEMOGRÁFICA \\
\hline Evolução do PIB & $\begin{array}{l}\text { Disponibilidade } \\
\text { tecnológica }\end{array}$ & $\begin{array}{l}\text { Regulamento da } \\
\text { concorrência }\end{array}$ & Estilos de vida & $\begin{array}{l}\text { Crescimento da } \\
\text { população }\end{array}$ \\
\hline Inflação & Tecnologia requerida & Leis ambientais & Moda & Migrações internas \\
\hline Taxa de juros & Direitos e patentes & Incentivos à indústria & Nível socioeconômico & Migrações externas \\
\hline Renda per capita & Complexidade & $\begin{array}{l}\text { Regulação sobre a } \\
\text { segurança }\end{array}$ & $\begin{array}{l}\text { Acessos aos meios } \\
\text { de comunicação }\end{array}$ & Estrutura familiar \\
\hline Nível de emprego & Tecnologia substitutivas & $\begin{array}{l}\text { Proteção ao } \\
\text { consumidor }\end{array}$ & Questões ambientais & \multirow{4}{*}{$\begin{array}{l}\text { Densidade } \\
\text { populacional }\end{array}$} \\
\hline Exportações & \multirow{3}{*}{$\begin{array}{l}\text { Maturidade } \\
\text { envolvida }\end{array}$} & Legislação sindical & \multirow{3}{*}{$\begin{array}{l}\text { Mudanças de hábitos } \\
\text { e de tradições }\end{array}$} & \\
\hline Evolução da indústria & & Legislação sobre o & & \\
\hline Taxa de câmbio & & & & \\
\hline
\end{tabular}

Fonte: adaptado de Leão et al. (2010)

Schwartz (2004) articula as seguintes etapas sucessivas para a elaboração de cenários: $1^{\mathrm{a}}$ ) identificar a questão central; $2^{\mathrm{a}}$ ) listar as forças-chave que influenciam o sucesso ou fracasso no ambiente local; $3^{\mathrm{a}}$ ) identificação das forças motrizes no macro ambiente que influenciam as forças-chave; $4^{\mathrm{a}}$ ) hierarquizar as forças-chave e forças motrizes por grau de importância e de incerteza, identificando os dois ou três fatores mais importantes e os mais incertos; $5^{\mathrm{a}}$ ) selecionar a lógica dos cenários, apontando as forças que os conduzem; $6^{\text {a }}$ ) encorpar os esqueletos dos cenários com os fatores-chave e tendências listados nas etapas dois e três; $7^{\text {a }}$ ) analisar as implicações dos cenários montados na etapa anterior, respondendo as seguintes questões: a) como está a decisão em cada cenário? b) quais vulnerabilidades serão reveladas? c) a decisão ou estratégia funciona em todos os cenários ou em qual? d) se o cenário desejado não acontecer, essa estratégia poderá se adaptar? E, $8^{\mathrm{a}}$ ) selecionar os indicadores iniciais na elaboração dos cenários e observar os sinais de aviso no presente que revelarão as tendências que estarão mais próximas ou não dos cenários elaborados.

Schoemaker (1995) aponta o seguinte modelo para o desenvolvimento de cenários: 1) Definir o Escopo: O primeiro passo é definir o quadro da análise (em termos de produtos, marcadores, áreas geográficas e tecnologias). Quais têm sido fontes passadas de incertezas e volatilidade? 2) Identificar os principais interessados: Quem vai ter interesse nestas questões? Quem será afetado por elas? Quem poderia influenciá-los? Interessados incluem clientes, fornecedores, concorrentes e assim por diante; 3) Identificar tendências básicas: Qual o grau de certeza que as tendências políticas, econômicas, sociais, tecnológicas e jurídicas afetam as questões identificadas na primeira eta- 
pa? 4) Identificar principais incertezas: Que eventos, cujos resultados são incertos, irão afetar significativamente as questões com que se está preocupado? 5) Construir temas para o cenário inicial: Depois de identificar tendências e incertezas, têm-se os principais ingredientes para a construção de cenários. Identificam-se cenários extremos, colocando todos os elementos positivos em um e todos os negativos na outra (positivo ou negativo é definido em relação à estratégia atual); 6) Verificar se há coerência e plausibilidade: Os cenários que foram elaborados provavelmente têm inconsistências internas; 7) Desenvolver cenários de aprendizagem: A partir deste processo de construção de cenários, alguns temas gerais devem surgir. O objetivo é identificar temas que têm importância estratégica e em seguida, orga- nizar os possíveis resultados e tendências em torno deles; 8) Identificar necessidades de pesquisa: Neste ponto, pode ser preciso fazer mais pesquisas para concretizar a compreensão das incertezas e tendências; 9) Desenvolver modelos quantitativos: Depois de ter completando a pesquisa adicional, deve-se reexaminar as consistências internas dos cenários e avaliar as interações através de um modelo quantitativo; 10) Evoluir para decisão sobre quais cenários: Finalmente, numa processo interativo, deve-se convergir para cenários que onde serão testadas as estratégias e gerar novas ideias.

Ao pesquisarem na literatura acadêmica, Boaventura, Costa e Fischmann (2005) identificaram os modelos de elaboração de cenários mais citados, os quais estão expostos no Quadro 5.

QUADRO 5: MÉTODOS DE ELABORAÇÃO DE CENÁRIOS IDENTIFICADOS NA LITERATURA ACADÊMICA

\begin{tabular}{|c|c|c|}
\hline MÉTODOS & ETAPAS & CARACTERISTICAS \\
\hline $\begin{array}{l}\text { SRI } \\
\text { International } \\
\text { (Stanford } \\
\text { Research } \\
\text { Institute). }\end{array}$ & $\begin{array}{l}\text { 1. Definição das decisões estratégicas que os cenários } \\
\text { deverão abordar; } 2 \text {. Identificação dos fatores-chave } \\
\text { de decisão; } 3 \text {. Análise das forças ambientais; } 4 \text {. } \\
\text { Desenvolvimento dos cenários lógicos; } 5 \text {. Descrição dos } \\
\text { cenários; } 6 \text {. Identificação das implicações estratégicas para } \\
\text { a tomada de decisão. }\end{array}$ & $\begin{array}{l}\text { - Lógica Intuitiva; } \\
\text { - Interatividade; } \\
\text { - Workshops. }\end{array}$ \\
\hline $\begin{array}{l}\text { Global Business } \\
\text { Network }\end{array}$ & $\begin{array}{l}\text { 1. Identificar a questão ou decisão central; } 2 \text {. Identificação } \\
\text { dos fatores-chave do ambiente local; } 3 \text {. Identificação das } \\
\text { forças motrizes do macro ambiente; } 4 \text {. Hierarquização dos } \\
\text { fatores chave por importância e incerteza; } 5 \text {. Seleção da } \\
\text { lógica dos cenários; } 6 \text {. Redação dos cenários; } 7 \text {. Análise } \\
\text { das implicações; } 8 \text {. Seleção dos indicadores iniciais e dos } \\
\text { sinais de aviso para monitoramento do futuro. }\end{array}$ & $\begin{array}{l}\text { - Aproximação com o modelo SRI } \\
\text { (Peter Schwartz, fundador da GBN } \\
\text { era consultor da SRI International); } \\
\text { - Lógica Intuitiva. }\end{array}$ \\
\hline Future Mapping & $\begin{array}{l}\text { Uso de workshops, com etapas bem definidas: } 1 \text {. Antes } \\
\text { - criação dos estados finais (quadro de uma indústria } \\
\text { em um ponto particular do tempo, escritos tipicamente } \\
\text { em conjuntos de quatro ou cinco) e dos eventos (uma } \\
\text { tendência pode ser desagregada em uma série de eventos); } \\
\text { 2. Explicitação do modelo mental vigente - cenários de } \\
\text { sabedoria convencional; } 3 \text {. Mapeamento dos estados finais; } \\
\text { participantes são divididos em grupos; } 4 \text {. Construção dos } \\
\text { cenários a apresentação para o grupo; } 5 \text {. Análise dos } \\
\text { pontos comuns e divergentes para os cenários; } 6 \text {. Escolha } \\
\text { do estado final mais desejável; } 7 \text {. Mapeamento da direção } \\
\text { estratégica. }\end{array}$ & $\begin{array}{l}\text { - O futuro é contingente e moldado } \\
\text { pela ação de vários participantes; } \\
\text { - Na maioria das indústrias, } \\
\text { esforços para conseguir vantagens } \\
\text { competitivas irão causar mudanças } \\
\text { estruturais; } \\
\text { - David Mason. }\end{array}$ \\
\hline
\end{tabular}




\begin{tabular}{|c|c|c|}
\hline MÉTODOS & ETAPAS & CARACTERISTICAS \\
\hline $\begin{array}{l}\text { Análise } \\
\text { Prospectiva }\end{array}$ & $\begin{array}{l}\text { 1. Análise do problema e delimitação do sistema; } 2 \text {. } \\
\text { Diagnóstico da empresa; } 3 \text {. Análise estrutural; } 4 \text {. Dinâmica } \\
\text { da empresa no ambiente; } 5 \text {. Cenários ambientais; } 6 \text {. } \\
\text { Identificação das estratégias; } 7 \text {. Avaliação das estratégias; } \\
\text { 8. Seleção das estratégias; } 9 \text {. Elaboração de planos de } \\
\text { ação e monitoramento da estratégia. }\end{array}$ & $\begin{array}{l}\text { - Aproximação entre análise } \\
\text { prospectiva e estratégia; } \\
\text { - Michel Godet. }\end{array}$ \\
\hline $\begin{array}{l}\text { Comprehensive } \\
\text { Situation } \\
\text { Mapping }\end{array}$ & $\begin{array}{l}\text { Duas etapas: 1. Fase divergente: a visão individual de cada } \\
\text { tomador de decisão sobre a natureza e estrutura da situação } \\
\text { estratégica é diagramada separadamente, sem a influência } \\
\text { da ideia dos outros participantes do processo; } 2 \text {. Fase } \\
\text { convergente: os participantes do processo interagem em um } \\
\text { debate com apresentações dos diagramas desenvolvidos } \\
\text { individualmente, análise das hipóteses mais e menos } \\
\text { importantes e uma possível consolidação das ideias. }\end{array}$ & $\begin{array}{l}\text { - Considera os impactos da } \\
\text { ocorrência simultânea e recíproca } \\
\text { das variáveis; } \\
\text { - Não atribui probabilidades; } \\
\text { - Mapeamento cognitivo com } \\
\text { a integração com um sistema } \\
\text { computadorizado. }\end{array}$ \\
\hline $\begin{array}{l}\text { Análise do } \\
\text { Impacto de } \\
\text { Tendências }\end{array}$ & $\begin{array}{l}\text { Três etapas: 1. Preparação: a) definição do foco - questões } \\
\text { que precisam ser respondidas para definir os limites dos } \\
\text { cenários a serem criados; b) mapeamento das forças } \\
\text { motrizes que têm maior capacidade de moldar o futuro do } \\
\text { setor. } 2 \text {. Desenvolvimento: a) construção do espaço do } \\
\text { cenário com a classificação dos vários estados futuros } \\
\text { em função das forças motrizes; b) seleção dos cenários } \\
\text { a serem detalhados; c) detalhamento dos cenários, } \\
\text { relacionando as tendências e os eventos necessários para } \\
\text { se chegar a cada um dos estados finais. 3. Documentação } \\
\text { e utilização: a) documentação, compreendendo quadros e } \\
\text { narrativas que descrevam a história representada em cada } \\
\text { cenário; b) comprovação das implicações de cada cenário } \\
\text { - quão diferentes serão as decisões sobre os negócios em } \\
\text { função de cada tipo de cenário. }\end{array}$ & $\begin{array}{l}\text { - Combina séries temporais } \\
\text { e econometria com fatores } \\
\text { qualitativos; } \\
\text { - Força o usuário a identificar } \\
\text { explicitamente os fatores de } \\
\text { impacto e avaliar tanto sua } \\
\text { probabilidade de ocorrência como } \\
\text { sua importância. }\end{array}$ \\
\hline $\begin{array}{l}\text { Decision } \\
\text { Strategies } \\
\text { International }\end{array}$ & $\begin{array}{l}\text { 1. Definição do escopo: definição do horizonte de tempo } \\
\text { ao qual os cenários se referem e definição do escopo de } \\
\text { análise, as matérias sobre as quais os cenários serão } \\
\text { elaborados. 2. Identificação dos principais interessados } \\
\text { (stakeholders).3. Identificação de tendências básicas: quais } \\
\text { tendências políticas, econômicas, sociais, tecnológicas, } \\
\text { legais e da indústria poderão afetar as matérias identificadas } \\
\text { no passo 1? 4. Identificação de incertezas-chave: } \\
\text { quais eventos, cujos resultados são incertos, afetarão } \\
\text { significativamente os assuntos-tema dos cenários? } 5 \text {. } \\
\text { Construção de temas de cenários iniciais: isso pode ser feito } \\
\text { identificando mundos extremos, relativamente à estratégia } \\
\text { corrente. } 6 \text {. Checagem da consistência e plausibilidade: } \\
\text { análise da compatibilidade das tendências com o horizonte } \\
\text { de tempo, combinação de resultados plausíveis e as reações } \\
\text { dos principais interessados, em termos de mudanças em } \\
\text { suas posições atuais. } 7 \text {. Desenvolvimento de cenários de } \\
\text { aprendizagem: a partir dos passos anteriores, alguns temas } \\
\text { relevantes podem emergir. } 8 \text {. Identificação de necessidades } \\
\text { de pesquisas adicionais: neste ponto, pode ser preciso } \\
\text { realizar pesquisas adicionais para aumentar o entendimento } \\
\text { das incertezas e tendências. } 9 \text {. Desenvolvimento de } \\
\text { modelos quantitativos: quantificar as consequências dos } \\
\text { vários cenários. 10. Desenvolver cenários de decisão: em } \\
\text { um processo iterativo, os elaboradores devem convergir } \\
\text { no sentido elaborar cenários que poderão ser usados para } \\
\text { testar a estratégia e gerar novas ideias. }\end{array}$ & $\begin{array}{l}\text { - Lógica Intuitiva; } \\
\text { - Paul Shoemaker. }\end{array}$ \\
\hline
\end{tabular}

Fonte: Da Silva, Spers e Wright (2012). 


\begin{tabular}{|c|c|c|}
\hline MÉTODOS & ETAPAS & CARACTERISTICAS \\
\hline $\begin{array}{l}\text { Análise do } \\
\text { Impacto de } \\
\text { Tendências }\end{array}$ & $\begin{array}{l}\text { Três etapas: 1. Preparação: a) definição do foco - questões } \\
\text { que precisam ser respondidas para definir os limites dos } \\
\text { cenários a serem criados; b) mapeamento das forças } \\
\text { motrizes que têm maior capacidade de moldar o futuro do } \\
\text { setor. } 2 \text {. Desenvolvimento: a) construção do espaço do } \\
\text { cenário com a classificação dos vários estados futuros } \\
\text { em função das forças motrizes; b) seleção dos cenários } \\
\text { a serem detalhados; c) detalhamento dos cenários, } \\
\text { relacionando as tendências e os eventos necessários para } \\
\text { se chegar a cada um dos estados finais. } 3 \text {. Documentação } \\
\text { e utilização: a) documentação, compreendendo quadros e } \\
\text { narrativas que descrevam a história representada em cada } \\
\text { cenário; b) comprovação das implicações de cada cenário } \\
\text { - quão diferentes serão as decisões sobre os negócios em } \\
\text { função de cada tipo de cenário. }\end{array}$ & $\begin{array}{l}\text { - Combina séries temporais } \\
\text { e econometria com fatores } \\
\text { qualitativos; } \\
\text { - Força o usuário a identificar } \\
\text { explicitamente os fatores de } \\
\text { impacto e avaliar tanto sua } \\
\text { probabilidade de ocorrência como } \\
\text { sua importância. }\end{array}$ \\
\hline $\begin{array}{l}\text { Decision } \\
\text { Strategies } \\
\text { International }\end{array}$ & $\begin{array}{l}\text { 1. Definição do escopo: definição do horizonte de tempo } \\
\text { ao qual os cenários se referem e definição do escopo de } \\
\text { análise, as matérias sobre as quais os cenários serão } \\
\text { elaborados. 2. Identificação dos principais interessados } \\
\text { (stakeholders).3. Identificação de tendências básicas: quais } \\
\text { tendências políticas, econômicas, sociais, tecnológicas, } \\
\text { legais e da indústria poderão afetar as matérias } \\
\text { identificadas no passo 1? } 4 \text {. Identificação de incertezas- } \\
\text { chave: quais eventos, cujos resultados são incertos, } \\
\text { afetarão significativamente os assuntos-tema dos cenários? } \\
\text { 5. Construção de temas de cenários iniciais: isso pode } \\
\text { ser feito identificando mundos extremos, relativamente } \\
\text { à estratégia corrente. } 6 \text {. Checagem da consistência e } \\
\text { plausibilidade: análise da compatibilidade das tendências } \\
\text { com o horizonte de tempo, combinação de resultados } \\
\text { plausíveis e as reações dos principais interessados, } \\
\text { em termos de mudanças em suas posições atuais. } 7 \text {. } \\
\text { Desenvolvimento de cenários de aprendizagem: a partir } \\
\text { dos passos anteriores, alguns temas relevantes podem } \\
\text { emergir. } 8 \text {. Identificação de necessidades de pesquisas } \\
\text { adicionais: neste ponto, pode ser preciso realizar pesquisas } \\
\text { adicionais para aumentar o entendimento das incertezas e } \\
\text { tendências. 9. Desenvolvimento de modelos quantitativos: } \\
\text { quantificar as consequências dos vários cenários. } 10 \text {. } \\
\text { Desenvolver cenários de decisão: em um processo iterativo, } \\
\text { os elaboradores devem convergir no sentido elaborar } \\
\text { cenários que poderão ser usados para testar a estratégia e } \\
\text { gerar novas ideias. }\end{array}$ & $\begin{array}{l}\text { - Lógica Intuitiva; } \\
\text { - Paul Shoemaker. }\end{array}$ \\
\hline
\end{tabular}

Fonte: Da Silva, Spers e Wright (2012).

O trabalho objetivando a construção de cenários tem seu valor na medida em que as organizações puderem elaborar respostas a suas perguntas categóricas sobre o futuro apontado pelos cenários (DA SILVA; SPERS;
WRIGHT, 2012). Para cada cenário haverá a necessidade de uma resposta apropriada, que será a Estratégia Robusta (SCHWARTZ, 2004). A Figura 1 ilustra a relação entre cenários múltiplos e a Estratégia Robusta. 
FIGURA 1: RELAÇÃO ENTRE CENÁRIOS MÚLTIPLOS E ESTRATÉGIA ROBUSTA

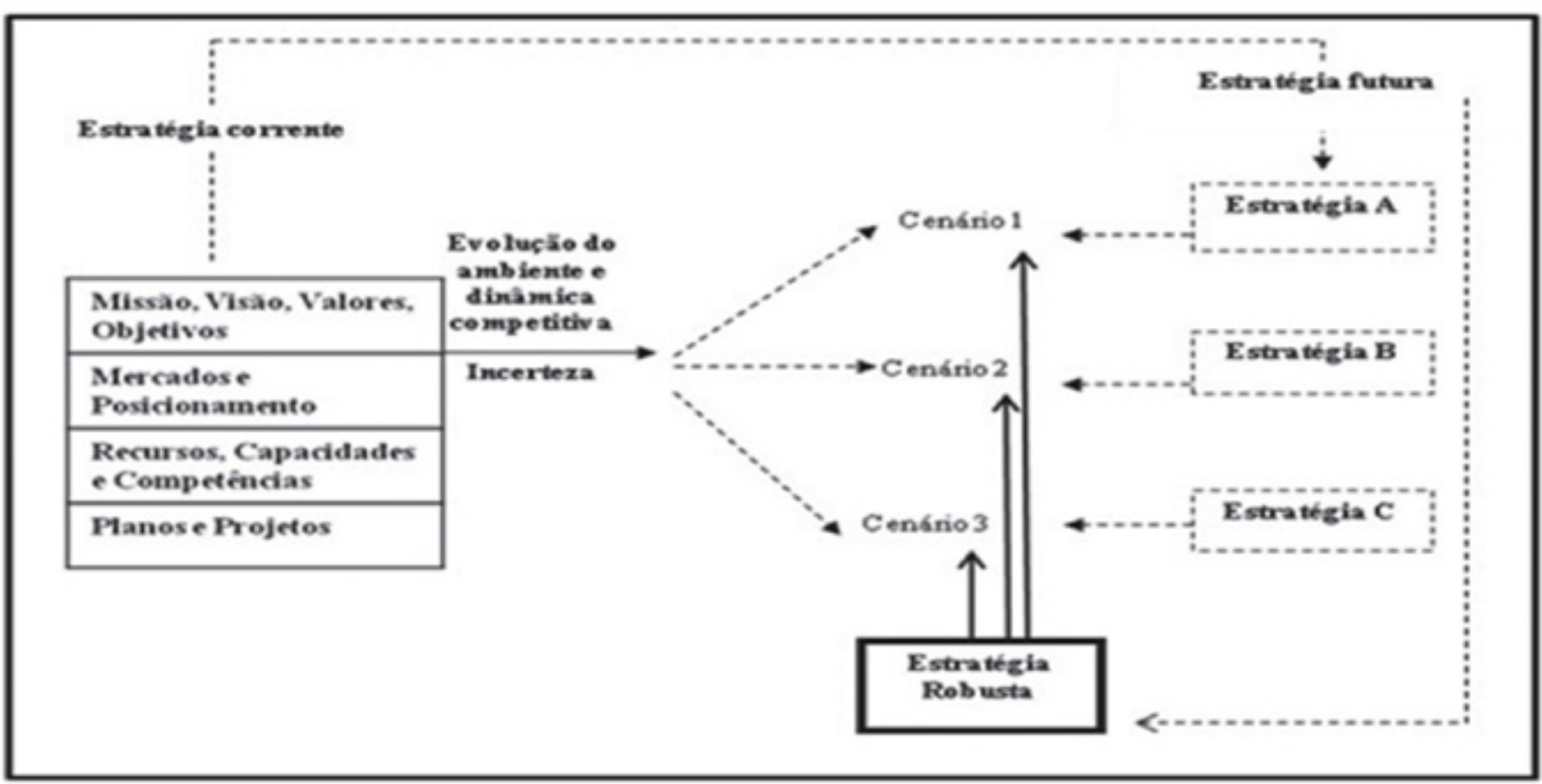

Fonte: Da Silva, Spers e Wright (2012)

Na Figura 1 percebe-se que, para a estratégia corrente, a organização baseia-se na análise das suas variáveis como Missão, Visão, Valores, Objetivos, Mercados, Posicionamento, Recursos, Capacidades, Competências, Planos e Projetos. Para a construção da estratégia futura é necessário aglutinar a decisão de como a organização objetiva se relacionar com o ambiente externo, considerando-se o aproveitamento das oportunidades e o enfrentamento das ameaças desse ambiente externo.

Portanto, faz-se necessário o diagnóstico externo e tem-se como pressuposto do planejamento que certas variáveis externas podem ser consideradas incertas. Uma possibilidade é a organização focar em um cenário único (Cenário 1 ou Cenário 2 ou Cenário 3) e elaborar uma estratégia única (Estratégia A ou Estratégia B ou Estratégia C) para atuar neste cenário. Outra alternativa é a organização considerar a possibilidade de ocorrência dos diferentes cenários e desenvolver uma estratégia robusta, aquela que seja capaz de funcionar nos diferentes cenários, seja no Cenário 1, no Cenário 2 e no Cenário 3 (DA SILVA; SPERS; WRIGHT, 2012).

\subsection{MÉTODOS DE ELABORAÇÃO DE CENÁRIOS ESTRATÉGICOS}

Bethlem (2009) articula que alguns métodos de criação de cenários possuem filosofia, etapas definidas e base con $\neg$ ceitual, tais como os métodos descritos por Porter (2004), Godet (2000) e Schwartz (2004). Em complemento a essa perspectiva, Wilson (2000) defende que ao serem analisados os diversos métodos na construção de cenários, quando se faz um detalhamento deles podem ser observadas a racionalidade e as características de cada um. Ribas (2008) catalogou uma relação de métodos por autor, técnica, estruturação, variáveis, probabilidades e horizonte na elaboração de cenários (Quadro 6). 
QUADRO 6: MÉTODOS DE ELABORAÇÃO DE CENÁRIOS

\begin{tabular}{|l|l|l|l|l|c|}
\hline \multicolumn{1}{|c|}{ AUTOR } & \multicolumn{1}{|c|}{ TÉCNICA } & ESTRUTURAÇÃO & VARIÁVEIS & PROBABILIDADES & HORIZONTE \\
\hline Asan e Asan (2007) & Impactos Cruzados & Exploratória & Event+Tend & Descritiva & Simples \\
Amara e Lipinski (1983) & Análise Morfológica & Exploratória & Eventos & Consistente & Multi \\
Becker (1989) & Análise Estrutural & Normativa & Event+Tend & Descritiva & Contínuo \\
Forrester (1968) & Dinâmica de & & & & \\
Sistemas & Exploratória & Event+Tend & Quantificada & Multi & \\
Godet (2000) & Análise Morfológica & Exploratória & Event+Tend & Consistente & Simples \\
Millett e Zelman (2005) & Simulação & Exploratória & Event+Tend & Consistente & Simples \\
Sapio (1995) & Impactos Cruzado & Exploratória & Event+Tend & Consistente & Simples \\
Scapolo e Miles (2006) & Impactos Cruzados & Normativa & Event+Tend & Descritiva & Simples \\
Weimer-Jehle (2006) & Impactos Cruzado & Exploratória & Event+Tend & Consistente & Simples \\
\hline
\end{tabular}

Fonte: Ribas (2008).

Pelo tipo de técnica utilizada na criação de cenários, Bradfield et al. (2005) as classificam como: brainstorm, clusters, dinâmica de sistemas, análise estrutural, análise dos atores, análise morfológica, Delphi, SMIC (sigla em francês para sistemas de impacto cruzado e matrizes), avaliação multicritério, impactos cruzados, impactos de tendências, Monte Carlo e simulação.

Para Godet (2000), o modo como os cenários são estruturados na sua elaboração, categorizam-se em exploratórias e normativas (ou antecipatórias). A primeira é um procedimento sequencial de causalidade entre variáveis na qual gradativamente ocorre a absorção de algumas dessas variáveis. A segunda detalha um modelo de situações futuras, em que o objetivo maior consiste na consistência interna dos cenários propostos.

Quanto à natureza das variáveis, Mitchell, Tydeman e Georgiades (1979) as consideram em eventos e tendências. Eventos são fenômenos categóricos que podem vir a ocorrer (ou não), em determinado momento no futuro, inesperadamente, influenciando o funcionamento do sistema. Tendências são uma série de eventos que se modificam gradual e sequencialmente ao longo do tempo e que causam mudanças contínuas no sistema.

As probabilidades são do tipo: a) consistentes: quando resultam de medição subjetiva e de aperfeiçoamento por meio de cálculos matemáticos ou simulação; b) quantificadas: quando estão relacionadas a uma escala subjetiva e não têm preocupação com a consistência; e c) descritivas: quando qualitativas, de gênero narrativo ou nominal (GODET, 1976).

Quanto ao horizonte, Becker (1989) dispõe o período de tempo em três tipos: a) simples: quando se limita a um tempo específico de análise e de descrição das situações alternativas até alcançar o seu final; b) multiperíodo: quando decorre da segmentação do intervalo de tempo, contendo diferentes cenários para cada momento; e c) contínuo: quando o tempo é tratado de maneira sem intervalos e os cenários se desenvolvem nesse período (RIBAS, 2008).

Como foi visto, há diversos métodos na elaboração de cenários, que diversificam de acordo com seus autores, técnica (Impactos Cruzados, Análise Morfológica, Análise Estrutural, citando alguns), estruturação (Exploratória e Normativa), variáveis (Event+Tend e Eventos), probabilidade (Descritiva, Consistente e Quantificada) e horizonte (Simples, Multi e Contínuo).

\section{DISCURSÃO DAS EVIDÊNCIAS TEÓRICO-CONCEITUAIS}

Nas bases pesquisadas, no período de 2000 a 2016, dentre as cinco palavras-chave strategic planning foi a que mais se destacou (representando $40,11 \%$ do total), seguida por Competitive strategy $(20,64 \%)$ e Competitiveness (16,98\%). Já no somatório das cinco palavras-chave, foi na base de pesquisa ProQuest onde foi encontrado um número mais expressivos de artigos (42.307), seguida pela Scopus (com 37.246 artigos). Tais informações podem ser observadas no Quadro 2. 
QUADRO 2: PALAVRAS CHAVE NAS BASES DE PESQUISA

\begin{tabular}{|l|c|c|c|c|c|c|c|}
\hline PALAVRAS CHAVE & EBSCO HOST & EMERALD & PROQUEST & SCOPUS & SCIENCE DIRECT & TOTAL & $\%$ \\
\hline Strategic scenarios & 63 & 40 & 3.353 & 420 & 57 & 3.933 & 3,90 \\
Competitive advantage & 1.905 & 806 & 10.728 & 3.312 & 385 & 17.136 & 16,98 \\
Strategic planning & 6.899 & 900 & 10.056 & 21.922 & 701 & 40.478 & 40,11 \\
Competitiveness & 4.813 & 1.939 & 1.654 & 9.234 & 893 & 18.533 & 18,37 \\
Competitive strategy & 619 & 1.107 & 16.516 & 2.358 & 228 & 20.828 & 20,64 \\
Total & 14.299 & 4.792 & 42.307 & 37.246 & 2.264 & 100.908 & 100,00 \\
\hline
\end{tabular}

Esse volume de publicações na Scopus e na ProQuest (Quadro 2) deu-se em função de serem, entre as cinco bases pesquisadas, as que possuem, respectivamente, em sua base de dados, cerca de 64 milhões de registros (o que inclui mais 21.548 títulos) e mais de 4 milhões de registros (incluindo mais de 4.560 títulos), conforme informações coletadas nos respecti- vos sites: https://www.scopus.com/freelookup/form/ author.uri; http://www.proquest.com/LATAM-PT/

Em contrapartida, quando foi realizada a pesquisa agrupando-se as palavras-chave strategic scenarios e competitive advantage, a quantidade de trabalhos que os relacionam foi de 5.257, enquanto que o número dos que associam as cinco palavras-chave juntas é de 2.256 (Quadro 3).

QUADRO 3: PALAVRAS CHAVE AGRUPADAS

\begin{tabular}{|c|c|c|c|c|c|c|}
\hline PALAVRAS CHAVE & EBSCO HOST & EMERALD & PROQUEST & SCOPUS & SCIENCE DIRECT & TOTAL \\
\hline $\begin{array}{l}\text { Strategic scenarios and } \\
\text { Competitive advantage }\end{array}$ & 108 & 847 & ---- & 3.988 & 314 & 5.257 \\
\hline $\begin{array}{l}\text { Strategic scenarios, } \\
\text { Competitive advantage, } \\
\text { Strategic planning, } \\
\text { Competitiveness and } \\
\text { Competitive strategy }\end{array}$ & 954 & 296 & 10 & 996 & ---- & 2.256 \\
\hline
\end{tabular}

Fonte: Elaboração dos autores.

As relações envolvendo strategic scenarios com competitive advantage revelam um número $33,66 \%$ maior (Quadro 3) do que o conceito chave stretegic scenarios sozinho (3.933, Quadro 2).

\subsection{CENÁRIOS E A VANTAGEM COMPETITIVA}

De acordo com os dados coletados nas bases de pesquisa, no período de 2000 a 2016, a associação das palavras chave: strategic scenarios com competitive advantage foram utilizadas em 5.257 artigos, enquanto que strategic scenarios foi encontrada em 3.933 artigos. Esses números sugerem que os autores dos artigos consideram que há uma relação entre a existência de cenários estratégicos como vantagem competitiva. Convém ressaltar que, em razão da concorrência globalizada, as organizações necessitam buscar a vantagem competitiva para agregar valor aos seus clientes e, ao mesmo tempo, crescerem e lucrarem (PORTER, 2004; PRAHALAD; HAMEL, 2005; VAN DER HEIJDEN, 2009; YEN-TSANG; CSILLAG; SIEGLER, 2012). A vantagem competitiva é a capacidade de criar mais valor do que os concorrentes e ocasionar retornos superiores sobre o investimento (KILLEN et al., 2012).

Por sua vez, enxergando a utilização de cenários como vantagem competitiva, Porter (2004), Prahalad e Hamel (2005) e Ribeiro (2012) assinalaram que a elaboração de planejamento utilizando cenários concede a empresa vantagem competitiva, em comparação com àquela que utiliza apenas o modo de 
planejamento tradicional, pois, no entender de Oliveira (2014), a construção de cenários fornece situações, critérios e medidas para que a empresa esteja mais bem preparada para enfrentar os desafios do futuro. Além do mais, a elaboração de cenários deixou de ser tratada como exercício de futurologia ou de mera previsão do futuro, já que, segundo Carlos et al. (2013), a formulação de cenários passou a se basear em métodos científicos, utilizando análises estatísticas de previsão.

No conceito de Oliveira (2014), cenários simulam realidades conjunturais que visam a preparação do futuro da empresa. No contexto de se organizar um planejamento usando cenários, Ribeiro (2012) observou há existência de cinco objetivos. Para: (1) a identificação de novas oportunidades no mercado; (2) o teste da estratégia em múltiplos cenários; (3) monitorar a execução da estratégia; (4) a identificação de mudanças no ambiente para selecionar as estratégias que deverão ser adaptadas ou alteradas; e, (5) a redução das incertezas com relação à capacidade da liderança em promover ajustes. Nessa perspectiva, Rojo (2010) sustentou que a elaboração de cenários constitui uma etapa importante que antecede ao planejamento estratégico e, de modo similar, Buytendijk, Hatch e Micheli (2010) e Postma e Liebl (2005) defenderam que a elaboração de cenários pode contribuir no desenho da estratégia da empresa.

Já Porter (2004) afirmou que cenário é uma visão internamente consistente do que o futuro pode ser e tem como principais funções a avaliação do que foi planejado, o apoio à elaboração de objetivos e de estratégias, a análise de alternativas, o estímulo à criatividade e a preparação para enfrentar descontinuidades.

\subsection{A ELABORAC̣̃̃O DE CENÁRIOS E MONITORAMENTO AMBIENTAL}

$\mathrm{Na}$ etapa inicial da elaboração de cenários, Paleo (2006) e da Silva, Spers e Wright (2012) destacaram a necessidade de que seja levado em consideração o conjunto de variáveis que atuam sobre o ambiente onde a organização está inserida. Porém, não há um modo único para a elaboração de cenários, como articularam Godet (2000) e Vianello e Teixeira (2012), mas diversos e que variam de acordo com o nível de complexidade.

Além de contribuir com a vantagem competitiva das empresas, Brandalise et al. (2012) e Ribas (2008) afirmaram que a criação de cenários é uma ferramen- ta que auxilia os gestores a refletirem acerca das consequências futuras das suas decisões no presente.

Leão et al. (2010) e Oliveira (2012) propuseram um norte para a elaboração de cenários: por meio de uma sequência cronológica de eventos, do presente até um momento futuro definido, constando nesse arcabouço as interações entre variáveis interdependentes (listadas no Quadro 4) e dimensões estratégicas relacionadas à empresa objeto de estudo.

Bontempo (2000) formulou 10 etapas sucessivas para a elaboração de cenários, já Schwartz (2004) apresentou um processo contendo 8 fases e Shoemaker (1995), expôs em sua pesquisa a existência de 10 etapas. Foi visto que no trabalho de Boaventura, Costa e Fischmann (2005) contém uma relação de 9 modelos de elaboração de cenários identificados na literatura (Quadro 5). Com efeito, pode ser observado nos estudos desses autores, pontos comuns em todos os modelos, os quais são: (i) analisar as variáveis externas do ambiente passado e presente; (ii) avaliar as variáveis internas da empresa: Missão, Visão, Valores, Objetivos, Mercados, Posicionamento, Recursos, Capacidades, Competências, Planos e Projetos; (iii) projetar tendências das variáveis ambientais, utilizando cálculos estatísticos e de probabilidade; (iv) planejar possíveis cenários, distinguindo-os em termos de probabilidade da ocorrência; e, (v) arquitetar estrategicamente as ações que a empresa adote para cada cenário, no contexto de ameaças, oportunidades, pontos fortes e pontos fracos, pois, conforme postulou Da Silva, Spers e Wright (2012), independentemente do modelo de elaboração de cenários a ser escolhido, ele só terá seu valor na medida em que as organizações puderem preparar no presente respostas às suas perguntas sobre o futuro apontadas pelos cenários. Assim, à medida que os cenários são construídos, Da Silva, Spers e Wright (2012) indicaram para que a empresa desenvolva a Estratégia Robusta, considerada como sendo aquela que é capaz de funcionar eficientemente nos diferentes cenários: Cenário 1, Cenário 2 ou Cenário 3 (Figura 1).

\subsection{MÉTODOS DE ELABORAÇÃO DE CENÁRIOS ESTRATÉGICOS}

Há diversos métodos na elaboração de cenários catalogados na literatura, os quais Wilson (2000) os diferenciou pelos critérios da racionalidade e das características de cada, sendo que, alinhado com essa perspectiva, Ribas (2008) diversificou esses métodos de acordo com: (i) seus autores - Asan e Asan 
(2007), Amara e Lipinski (1983), Becker (1989), Forrester (1968), Godet (2000), Millett e Zelman (2005), Sapio (1995), Scapolo e Miles (2006) e Weimer-Jehle (2006); (ii) a técnica (Impactos Cruzados, Análise Morfológica, Análise Estrutural, citando alguns); (iii) o tipo de estruturação (Exploratória e Normativa), variáveis (Event+Tend e Eventos), probabilidade (Descritiva, Consistente e Quantificada); e, (iv) o horizonte (Simples, Multi e Contínuo).

Quanto à técnica utilizada para a concepção de cenários, ela pode ser classificada como: brainstorm, clusters, dinâmica de sistemas, análise estrutural, análise dos atores, análise morfológica, Delphi, SMIC (sigla em francês para sistemas de impacto cruzado e matrizes), avaliação multicritério, impactos cruzados, impactos de tendências, Monte Carlo e simulação (BRADFIELD et al., 2005). Godet (2000) definiu que o modo como os cenários são estruturados na sua elaboração, classificam-se em exploratórias e normativas (ou antecipatórias). No que tange à natureza das variáveis, Mitchell, Tydeman e Georgiades (1979) os qualificou em eventos e tendências. Godet (1976), especificou que as probabilidades são do tipo consistentes ou quantificadas. No que se refere ao horizonte de tempo, Becker (1989) elencou em simples, multiperíodo e contínuo.

\section{CONSIDERAÇÕES FINAIS}

Este estudo objetivou pesquisar como o tema "cenários estratégicos" vem sendo trabalhado na literatura. $\mathrm{O}$ referencial teórico que discorre sobre o conceito, métodos para a elaboração de cenários e o desenvolvimento da vantagem competitiva para a organização que utiliza estrategicamente a criação de cenários, mostra que o objetivo principal dos cenários não é o de se ter a certeza absoluta sobre o que prevalecerá no futuro, uma vez que a conjuntura futura será moldada no transcorrer do tempo presente.

$\mathrm{Na}$ elaboração de cenários leva-se em consideração a análise das variáveis independentes (econômica, tecnológica, política, sócio cultural e demográfica), independentemente do modelo que venha a ser utilizado na sua criação: Schwartz (2004), Schoemaker (1995), SRI International, Global Business Network, Future Mapping, Battelle Memorial Institute, Análise Prospectiva, Comprehensive Situation Ma- pping, Análise do Impacto de Tendências, Decision Strategies International, Asan e Asan (2007), Amara e Lipinski (1983), Becker (1989), Forrester (1968) e Godet (2000), dentre outros expostos neste trabalho. No geral, esses modelos sugerem a criação de até três cenários (Otimista, Realista e Pessimista) para que a organização desenvolva uma Estratégia Robusta que seja capaz de funcionar eficientemente e obter os resultados esperados em qualquer um.

Da Silva, Spers e Wright (2012) e Huss (1988) salientaram que conhecer de antemão possíveis futuros favorece aos gestores das empresas para estarem mais bem preparados para definir estratégias e lidar com as incertezas e riscos deste ambiente em mudança e a criação de cenários constitui uma etapa importante que antecede ao da elaboração do planejamento estratégico.

Assim, buscou-se investigar a pesquisa acadêmica que aborda a elaboração e o uso de cenários, demonstrando essa ferramenta como elemento potencializador na formulação das estratégias. Para a construção da estratégia futura, é necessário a organização alinhar suas variáveis (Missão, Visão, Valores, Objetivos, Mercados, Posicionamento, Recursos, Capacidades, Competências, Planos e Projetos) com a decisão de como objetiva se relacionar com o ambiente externo.

Chegou-se ao entendimento que é obtida uma vantagem competitiva em relação às tradicionais formas de planejamento, quando a organização utiliza o planejamento por cenários, uma vez que os cenários são elaborados para auxiliar na formulação das estratégias.

Para pesquisas futuras, sugere-se a criação de modelos de elaboração de cenários que incluam os integrantes da cadeia de suprimentos, pois a gestão da cadeia de suprimentos tem sido considerada um dos principais fatores condicionantes da competitividade das organizações (GUERREIRO; BIO; MENDEL, 2011; MENTZER et al., 2001; PRAJOGO; SOHAL, 2013; TAYLOR, 2008). Ademais, em um ambiente globalizado, as empresas já constataram que a concorrência já não dependente apenas das capacidades das empresas individuais, mas da capacidade de uma cadeia de suprimentos totalmente integrada entre seus membros (MIN; ZHOU, 2002; PRAJOGO; SOHAL, 2013; SCAVARDA; SCAVARDA, 2004; CHOON TAN, LYMAN; WISNER, 2002). 


\section{REFERÊNCIAS}

ABREU, A. de; ALCANTARA, R. L. C. A gestão de pessoas e a estrutura organizacional sob a perspectiva da cadeia de suprimentos. Reuna, v. 19, n. 1, p. 67-88, 2014.

ALVES, C. G. F.; OLIVEIRA, M. A. Rede de interações internacionais: produção científica sobre inovação e centros de pesquisa. Revista ADM.MADE, ano 14, v.18, n.2, p.75-98, 2014.

AMARA, R.; LIPINSKI, A. Business planning for an uncertain future: scenarios \& strategies. Oxford: Pergamon Press, 1983.

ASAN, S. S.; ASAN, U. Qualitative cross-impact analysis with time consideration. Technological Forecasting and Social Change, v. 74, n. 5, p. 627-644, 2007.

BECKER, H. S. Developing and using scenarios: assisting business decisions. Journal of Business \& Industrial Marketing, v. 4, n. 1, p. 61-70, 1989.

BETHLEM, A. Estratégia empresarial: conceitos, processo e administração estratégica. 6. ed. São Paulo: Atlas, 2009.

BLOIS, H. D.; SOUZA, J. C. Cenários prospectivos e a dinâmica de sistemas: proposta de um modelo para o setor calçadista. Revista de Administração de Empresas, v. 48, n. 3, p. 35-45, 2008.

BOAVENTURA, J. M. G.; COSTA, B. K.; FISCHMANN, A. A. Cenários: metodologias e métodos de construção. In: COSTA, B. K.; DE ALMEIDA, M. I. R. de (Org.). Estratégia: direcionando negócios e organizações. São Paulo: Atlas, 2005.

BONTEMPO, M. T. Análise comparativa dos métodos de construção de cenários estratégicos no planejamento empresarial. (Dissertação de Mestrado em Controladoria e Contabilidade)- Faculdade de Economia, Administração e Contabilidade da Universidade de São Paulo, São Paulo, 2000.

BRADFIELD, R. et al. The origins and evolution of scenario techniques in long range business planning. Futures, v. 37, p. 795-812, 2005.

BRANDALISE, L. T. et al. Simulação de cenários e formulação de estratégias competitivas: o caso do atacado liderança. Revista Gestão \& Tecnologia, v. 12, n. 3, p. 223-257, 2012.

BUYTENDIJK, F.; HATCH, T.; MICHELI, P. Scenario-based strategy maps. Business Horizons, v. 53, n. 4, p. 335-347, 2010.

CARLOS, M. G. O. et al. Era uma vez o futuro! - o que se tem produzido no século XXI sobre cenários na academia brasileira de administração? In: Encontro da ANPAD, 37, Rio de Janeiro, 2013. p. 1-16.

CENTRAL INTELLIGENCE AGENCY. O relatório da CIA: como será o mundo em 2020. São Paulo: Ediouro, 2006.

CHOON TAN, K.; LYMAN, S. B.; WISNER, J. D. Supply chain management: a strategic perspective. International Journal of Operations \& Production Management, v. 22, n. 6, p. 614-631, 2002.

COATES, J. F. Scenario planning. Technological Forecasting and Social Change. Nova York, v. 65, n. 1, p. $115-123,2000$. 


\section{raUnP}

COSTA, B. K. et al. Adequação e uso de cenários prospectivos: um estudo no órgão municipal de turismo de Natal. Turismo - Visão e Ação, v. 9, n.1, p. 7-17, 2007.

DA SILVA, A. T. B.; SPERS, R. G.; WRIGHT, J. T. C. A elaboração de cenários na gestão estratégica das organizações: um estudo bibliográfico. Revista de Ciências da Administração, v. 12, n. 32, p. 21-34, 2012.

FALLER, L. P.; DE ALMEIDA, M. I. R. Planejamento por cenários: preparando pequenas empresas do varejo de móveis planejados para um futuro competitivo. Revista de Administração, v.49, n.1, p.171-187, 2014.

FERREIRA, M. A bibliometric study on Ghoshal's managing across borders. Multinational Business Review, v. 19, n. 4, p. 357-375, 2011.

FORRESTER, J. W. Industrial Dynamics: after the first decade. Management Science, v. 14, n. 7, p. 398-415, 1968.

FOTR, J. et al. Scenarios, their concept, elaboration and application. Baltic Journal of Management, v. 10, n. 1, p. 73-97, 2015.

FRIEDMAN, G. Os próximos 100 anos: uma previsão para o século XXI. Rio de Janeiro: Best Seller, 2009.

GODET, M. The art of scenarios and strategic planning: tools and pitfalls. Technological Forecastingand Social Change, v. 65, n. 1, p. 3-22, 2000.

Scenarios of air transport development to 1990 by SMIC 74: a new cross-impact method. Technological Forecasting and Social Change, v. 9, p. 279-288, 1976.

GUERREIRO, R.; BIO, S. R.; MENDEL, S. F. Logística integrada, gestão da cadeia de suprimentos mensuração de custos e resultados logísticos: um estudo com empresas brasileiras. Advances in Scientific and Applied Accounting, v. 4, n. 1, p. 73-100, 2011.

GUGAN, A. Successful scenario planning. JISC infoNet. 2008. Disponível em:<www.jisc.ac.uk/publications $>$ Acesso em 02/07/2016.

HARZING, A.; ALAKANGAS, S. Google Scholar, Scopus and the Web of Science: a longitudinal and crossdisciplinary comparison. Scientometrics, v. 106, n. 2, p. 787-804, 2016.

HUSS, W. R. A move toward scenario analysis. International Journal of Forecasting. Nova York, v. 4, n. 3, p. 377-388, 1988.

JAMES, H. The end of globalization: lessons from the great depression. 4. th. Cambridge MA: Harvard University Press, 2002.

KILLEN, C. et al. Advancing project and portfolio management research: Applying strategic management theories. International Journal of Project Management, v. 30, n. 5, p.525-538, 2012.

LEÃO, P. R. C. et al. A relevância da metodologia de cenários para instituições financeiras em momentos de crise. Future Studies Research Journal, v. 2, n. 2, p. 38-55, 2010.

MAGALHÃES, R. C.; BARP, A. R. B. Inovações metodológicas para construção de cenários estratégicos em bacias hidrográficas. Revista de Administração e Inovação, v. 11, n. 3, p. 200-226, 2014.

MENTZER, J. T. et al. Defining supply chain management. Journal of Business Logistics, v. 22, n. 2, p. 1-25, 2001. 
MICHEL, M. H. Metodologia e pesquisa cientifica em ciências sociais. 3. ed. atual. e ampl. São Paulo: Atlas, 2015.

MILLETT, S. M.; ZELMAN, S. T. Scenario analysis and a logic model of public education in Ohio. Strategy \& Leadership, v. 33, n. 2, p. 33-40, 2005.

MIN, H.; ZHOU, G. Supply chain modeling: past, present and future. Computers \& Industrial Engineering, v. 43, n. 1, p. 231-249, 2002.

MITCHELL, R. B.; TYDEMAN, J.; GEORGIADES, J. Structuring the future: application of a scenariogeneration procedure. Technological Forecasting and Social Change, v. 14, n. 4, p. 409-428, 1979.

MÜLLER, C. J. Modelo de gestão integrando planejamento estratégico, sistemas de avaliação de desempenho e gerenciamento de processos (MEIO - Modelo de Estratégia, Indicadores e operações). Tese (Doutorado em Engenharia da Produção)- Escola de Engenharia, Universidade Federal do Rio Grande do Sul, Porto Alegre, 2003.

OLIVEIRA, D. P. R. Planejamento estratégico: conceitos, metodologia e práticas. 32. ed. São Paulo: Atlas, 2014.

. Estratégia empresarial e vantagem competitiva: como estabelecer, implementar e avaliar. 8. ed. São Paulo: Atlas, 2012.

ONKAL, D.; SAYIM, K. Z.; GÖNÜL, M. S. Scenarios as channels of forecast advice. Technological Forecasting \& Social Change, v. 80, n. 4, p. 772-788, 2013.

PALEO, O. Prospecção do futuro através da construção de cenários. Think, v. 4, n. 2, p. 60-66, 2006.

PIZZINATTO, N. K. et al. Tendências estratégicas e o perfil do gestor. Revista de Administração da UNIMEP, v. 8, n. 3, p. 239-255, 2010.

PORTER, M. E. Estratégia competitiva: técnicas para análise de indústrias e da concorrência. 2. ed. Rio de Janeiro: Elsevier, 2004.

POSTMA, T. J.B.M.; LIEBL, F. How to improve scenario analysis as a strategic management tool? Technological Forecasting and Social Change, v. 72, p. 161-173, 2005.

PRAHALAD, C. K.; HAMEL, G. Competindo pelo futuro: estratégias inovadoras para obter o controle do seu setor e criar os mercados de amanhã. 24. ed. Rio de Janeiro: Elsevier, 2005.

PRAJOGO, D.; SOHAL, A. Supply chain professionals. International Journal of Operations \& Production Management, v. 33, n. 11-12, p. 1532-1554, 2013.

RIBAS, J. R. Combinando intuição e objetividade na construção de cenários alternativos. Revista de Gestão USP, v. 15, n. 4, p. 1-17, 2008.

RIBEIRO, M. P. M. Planejamento por cenários: uma ferramenta para a era do conhecimento. Revista Intersaberes, v. 1, n. 1, p. 186-202, 2012.

ROJO, C. A. Simulação de cenários e a estratégia nos negócios. CAP Accounting and Management, v. 1, n. 1, p. 37-44, 2010.

SAMPIERI, R. H.; COLLADO, C. F.; LUCIO, P. B. Metodologia de pesquisa. 5. ed. São Paulo: Penso, 2013. 
SAPIO, B. SEARCH (Scenario evaluation and analysis through repeated cross impact handling): a new method for scenario analysis with an application to the Videotel service in Italy. International Journal of Forecasting, v. 11, n. 1, p. 113-131, 1995.

SCAPOLO, F.; MILES, I. Eliciting experts' knowledge: a comparison of two methods. Tech44 
nological Forecasting and Social Change, v. 73, n. 6, p. 679-704, 2006.

SCAVARDA, L. F.; SCAVARDA, A. J. Viabilizadores para a supply chain management. Revista de Administração FACES Journal, v. 3, n. 2, p. 11-25, 2004.

SCHENATTO, F. J. A. et al. Análise crítica dos estudos do futuro: uma abordagem a partir do resgate histórico e conceitual do tema. Gestão e Produção, São Carlos, v. 18, n. 4, p. 739-754, 2011.

SCHOEMAKER, P. J. H. Scenario planning: a tool for strategic thinking. Sloan Management Review, v. 36, n. 2, p. 25-40, 1995.

SCHWARTZ, P. A arte da visão de longo prazo: planejando o futuro em um mundo de incertezas. São Paulo: Best Seller, 2004.

SERRA, F.; TORRES, M. C. S.; TORRES, A. P. Administração estratégica: conceitos, roteiro prático e casos. Rio de Janeiro: Reichmann \& Affonso Editores, 2004.

SNYDER, C. A case study of a case study: analysis of a robust qualitative research methodology. The Qualitative Report, v. 17, p. 1-21, 2012.

TAYLOR, D. A. Logística na cadeia de suprimentos: uma perspectiva gerencial. São Paulo: Pearson AddisonWesley, 2008.

TRANFIELD, D.; DENYER, D.; SMART, P. Towards a methodology for developing evidence-informed management knowledge by means of systematic review. British Journal of Management, v. 14, p. 207-222, 2003.

VAN DER HEIJDEN, K. Planejamento por cenários: arte da conversação estratégica. 2. ed. Porto Alegre: Bookman, 2009.

VIANELLO, J. M.; TEIXEIRA, J. P. Modelagem da incerteza nos valores de investimentos de projetos do setor petrolífero. Tecnologias de Administração e Contabilidade-TAC/ANPAD, Rio de Janeiro, v. 2, n. 2, art. 2, p. 127-152, 2012.

VICENTINE, C. M. Inovação e administração estratégica para os novos cenários competitivos do século XXI. REBRAE-Revista Brasileira de Estratégia, v. 2, n. 3, p. 225-232, 2009.

WEBSTER, J.; WATSON, J. T. Analyzing the past to prepare for the future: writing a literature review. MIS Quarterly \& The Society for Information Management, v. 26, n. 2, p. 13-23, 2002.

WEIMER-JEHLE, W. Cross-impact balances: a system-theoretical approach to cross-impact analysis. Technological Forecasting and Social Change, v. 73, n. 4, p. 334-361, 2006.

WILSON, I. From scenario thinking to strategic action. Technological Forecasting and Social Change, v. 65 , n. 1, p. 23-29, 2000.

WRIGHT, J. T. C.; SPERS, R. G. O país no futuro: aspectos metodológicos e cenários. Estudos Avançados, v. 20, n. 56, p. $13-28,2006$.

WRIGHT, P.; KROLL, M. J.; PARNELL, J. Administração estratégica: conceitos. São Paulo: Atlas, 2009.

YEN-TSANG, C.; CSILLAG, J. M.; SIEGLER, J. Theory of reasoned action for continuous improvement capabilities: a behavioral approach. Revista de Administração de Empresa, v. 52, n. 5, p. 546-564, 2012. 